\title{
AVALIAÇÃO QUÍMICA DO EXPERIMENTO TERRA PRETA NOVA: TECNOLOGIA SOCIAL APLICADA EM SALVAGUARDA AO MEIO AMBIENTE
}

\section{CHEMICAL EVALUATION OF THE TERRA PRETA NOVA EXPERIMENT: SOCIAL TECHNOLOGY APPLIED IN SAFEGUARD TO THE ENVIRONMENT}

\author{
Thalita Alves Cirilo Batista ${ }^{1}$; Maura Gabriela da Silva Brochado ${ }^{2}$; Paulo Cerqueira Júnior ${ }^{3}$; \\ Milena Carvalho de Moraes ${ }^{4}$; José Francisco Berredo ${ }^{5}$
}

DOI: https://doi.org/10.31692/978-65-991061-7-0.153-162

\section{RESUMO}

Os solos de Terra Preta Arqueológica (TPA) são provenientes de atividades antrópicas pretéritas de manejo e uso da terra, desenvolvidas na região amazônica, cujo legado resultante foram esses solos altamente férteis, estáveis e resistentes, diferentes dos solos da região. As características inerentes às TPA's são relevantes para o contexto agrícola e socioambiental, por ser um produto de inovação tecnológica com o foco de melhoria de produção de alimentos, no quesito qualidade, o qual atua diretamente na diminuição de impactos ambientais. Desta forma, a reprodutibilidade desses solos gerou o experimento de tecnologia social: "Terra Preta Nova TPN", o qual se desenvolve a 14 anos, na região de Tailândia - PA, o experimento é composto por 17 tratamentos, incluindo a parcela controle, com misturas variadas de carvão, resíduo de lâmina triturada, pó de serra, ossos e açougue, com delineamento experimental em bloco totalmente casualizado, com quatro repetições para cada tratamento. O objetivo do trabalho foi avaliar quimicamente a evolução do experimento TPN. As análises químicas foram realizadas na Universidade Federal de Viçosa, seguindo metodologia padrão do laboratório de análise química de solo, tecido vegetal e fertilizante. Foram avaliados os parâmetros químicos $\mathrm{pH}, \mathrm{H}+\mathrm{Al}, \mathrm{SB}, \mathrm{t}, \mathrm{T}, \mathrm{V}, \mathrm{m}, \mathrm{P}$-rem e a disponibilidade dos elementos $\mathrm{P}$, $\mathrm{K}, \mathrm{Mg}, \mathrm{Ca}$ e $\mathrm{Al}$ nas parcelas referentes a dois períodos chuvosos nos anos de 2004 e 2011, com a finalidade de avaliar qual o tratamento mais adequado em termos de composição química e atributos nutricionais para o solo. Os resultados demonstraram o tratamento da parcela 14 como o melhor avaliado em relação aos demais, devido ao aumento na maioria dos atributos analisados. Entretanto, mais estudos de caracterização são necessários para avaliar o experimento TPN de forma mais completa.

Palavras-Chave: Tecnossolos, arqueoantrossolos, estudo químico.

\begin{abstract}
\footnotetext{
${ }^{1}$ Agronomia, Universidade Federal Rural da Amazônia, batista05ab@gmail.com

2 Agronomia, Universidade Federal Rural da Amazônia, Maurabrochado@gmail.com

${ }^{3}$ Docente, Universidade Federal Rural da Amazônia, pauloest16@gmail.com

${ }^{4}$ PCI, Museu Paraense Emílio Goeldi, milenamoraes@museu-goeldi.br

${ }^{5}$ Doutor, Museu Paraense Emílio Goeldi, berredo@museu-goeldi.br
}

The soils of the Archaeological Black Earth (ABE) are are derived from previous anthropic activities of land use and management developed in the Amazon region, whose resulting legacy was these highly fertile, stable and resistant soils, different from the soils of the region. The characteristics inherent to TPA's are relevant to the agricultural and socio-environmental context, as it is a product of technological innovation focused on improving food production, in terms of quality, which acts directly in the reduction of environmental impacts. In this way, the reproducibility of these soils generated the experiment of social technology: "Terra Preta Nova - TPN", which develops at 14 years in the region of Tailandia - PA, the experiment is composed of 17 treatments, including the control plot, with mixed mixtures of charcoal, 
shredded residue, saw dust, bones and butchery, with a completely randomized block design with four replicates for each treatment. The objective of this work was to chemically evaluate the evolution of the TPN experiment. The chemical analyzes were carried out at the Federal University of Viçosa, following the standard methodology of the laboratory of chemical analysis of soil, plant tissue and fertilizer. The chemical parameters $\mathrm{pH}, \mathrm{H}+\mathrm{Al}, \mathrm{SB}, \mathrm{t}, \mathrm{T}, \mathrm{V}$, $\mathrm{m}, \mathrm{P}$-rem and the availability of the elements $\mathrm{P}, \mathrm{K}, \mathrm{Mg}, \mathrm{Ca}$ and $\mathrm{Al}$ were evaluated in the plots referring to two rainy periods in the years 2004 and 2011, with the purpose of evaluating the most appropriate treatment in terms of chemical composition and nutritional attributes for the soil. The results showed the treatment of plot 14 as the best evaluated in relation to the others, due to the increase in most attributes analyzed. However, further characterization studies are needed to evaluate the TPN experiment more fully.

Keywords: Technosoils, archaeoantrosoils, chemical study.

\section{INTRODUÇÃO}

As Terras Pretas Arqueológicas ocorrem frequentemente na Região Amazônica, especialmente na parte brasileira. Localmente são conhecidas como Terra Preta (TP), Terra Preta de Índio (TPI), ou Terra Preta Arqueológica/Antrópica (TPA), e resultam de profundas modificações, originadas por atividades antrópicas, incluindo adição de material orgânico, cerâmico, resíduos domésticos, irrigação e/ou agricultura (COSTA et al., 2013; TEIXEIRA et al., 2009).

A Terra Preta Arqueológica (TPA) é um vestígio comum em sítios arqueológicos amazônicos. As modificações antrópicas pelas quais as TPA's foram submetidas resultaram em efeitos positivos, com relação às propriedades de estabilidade da matéria orgânica e ao elevado teor de componentes organometálicos, contribuindo, para uma melhor qualidade desse sistema (KERN, 1996).

Além da elevada fertilidade as TPA's contrastam com os demais solos da região Amazônica (adjacentes) pela evidente coloração escura, incorporação de ossos, elevada capacidade de troca catiônica, matéria orgânica recalcitrante, horizonte A antrópico espesso, altos teores de P, Ca, K, Mn, Mg e C orgânico (GLASER et al., 2001; GERMAN, 2003; NOVOTNY et al., 2009; COSTA; COSTA; KERN, 2013). O elevado nível de matéria orgânica em TPA's pode ser atribuído, ao fato, de que eles foram formados no passado durante a ocupação humana destes locais (KERN, 1996; SILVEIRA et al., 2002).

A reprodutibilidade dos solos de Terra Preta Arqueológica (TPA) é de grande importância do ponto de vista científico, econômico e ambiental, pois as características peculiares das TPAs, que possam ser reproduzidas no substrato Terra Preta Nova (TPN) principalmente com relação à fertilidade, promovem solos altamente produtivos e estáveis, favorecendo a agricultura de modo geral, principalmente se adotada na agricultura familiar. 
O experimento TPN é uma tecnologia social desenvolvida pela Dra. Dirse Kern, pesquisadora do Museu Paraense Emílio Goeldi, e vem como uma solução voltada para o reaproveitamento de materiais de descarte, para a utilização como substrato do solo. $\mathrm{O}$ experimento é a reprodução de um solo mais fértil, com as características do solo de TPA. Dessa forma, não se trata de um adubo ou compostagem, mas um produto gerado a partir do aproveitamento econômico dos resíduos de madeira, como alternativa de minimizar os problemas socioambientais.

O experimento TPN possui interface com os objetivos globais estabelecidos para ODS 2 , que pretendem até 2030, dobrar a produtividade agrícola e a renda dos pequenos produtores de alimentos, particularmente das mulheres, povos indígenas, agricultores familiares, pastores e pescadores, inclusive por meio de acesso seguro e igual à terra, outros recursos produtivos e insumos, conhecimento, serviços financeiros, mercados e oportunidades de agregação de valor e de emprego não agrícola. Além de, garantir sistemas sustentáveis de produção de alimentos e implementar práticas agrícolas resilientes, que aumentem a produtividade e a produção, que ajudem a manter os ecossistemas, que fortaleçam a capacidade de adaptação às mudanças climáticas, às condições meteorológicas extremas, secas, inundações e outros desastres, e que melhorem progressivamente a qualidade da terra e do solo.

O experimento conta com 17 tipos de tratamentos para o solo, em misturas contendo Carvão, Resíduos de pó de serra, Resíduos de lâmina triturada, Resíduos de ossos Sangue e gordura animal. A composição mineralógica do solo, no qual, esses tratamentos serão misturados carrega uma grande importância, pois os minerais presentes no mesmo, podem reagir de diferentes formas com a adição dos materiais de naturezas diversas. Ao se agregarem com os materiais adicionados, podem ocorrer reações que levaram a gênese de novos minerais. Dependendo do mineral formado, pode haver uma otimização na produtividade do solo em diferentes culturas.

Neste contexto, o trabalho analisou quimicamente as amostras de TPN provenientes dos anos de 2004 e 2011, para avaliar a evolução do experimento e qual o tratamento mais adequado em termos de qualidade de solo.

\section{FUNDAMENTAÇÃO TEÓRICA}

A Terra Preta Nova é uma tecnologia de suma importância dentro de um caráter ambiental, econômico e social. Ela utiliza rejeitos de açougues e serrarias, com um objetivo de otimizar a fertilidade de diferentes tipos de solos, dessa forma esses rejeitos, que antes eram simplesmente descartados no meio ambiente, passam a ser aproveitados de uma forma que traz 
benefícios para a natureza e a agricultura. As serrarias produzem toneladas de pó de serra em meio a suas atividades, que são descartados no meio ambiente levando a ocorrência de problemas ambientais como queimadas por exemplo, que em ambientes de clima muito quente, podem ser conduzidas pelo produto descartado. Outra problemática associada a questões ambientais é a contaminação da água por resíduos bovinos, como gordura, sangue, e outros, advindos de açougues e abatedouros que devido a falta de uso para esses materiais, eles acabam sendo muitas vezes descartados em rios. Rejeitos dessa natureza também são lançados em meio terrestre, levando a proliferação de animais como roedores no local. A TPN agrega esses materiais a solos, e os mesmos reagem de uma forma que torna esse solo fértil, sendo assim eles podem ser utilizados no lugar de determinados adubos e/ou fertilizantes, sendo mais viáveis economicamente gerando benefício direto principalmente aos pequenos agricultores.

\section{METODOLOGIA}

O experimento Terra Preta Nova foi implantado no ano de 2004, na área de servidão da linha Tucurui/Albrás, coordenadas $0257^{\prime}$ 021'’S e $04857^{\prime}$ 21'’WGr, localizado no município de Tailândia - PA. Os solos dessas parcelas foram coletados no período seco e chuvoso de cada ano para fins de monitoramento químico, entretanto, para o trabalho foram selecionadas somente os anos de 2004 e 2011 do período chuvoso, onde foram analisados os parâmetros de $\mathrm{pH}, \mathrm{H}+\mathrm{Al}, \mathrm{SB}, \mathrm{t}, \mathrm{T}, \mathrm{V}, \mathrm{m}$, P-rem e a disponibilidade dos elementos $\mathrm{P}, \mathrm{K}, \mathrm{Mg}$, $\mathrm{Ca}$ e Al. As análises foram realizadas na Universidade Federal de Viçosa, seguindo metodologia padrão do laboratório de análise química de solo, tecido vegetal e fertilizante. Para o tratamento de dados foi utilizado o programa $\mathrm{R}$ de estatística.

No experimento, foram inicialmente implantados 17 tratamentos com quatro repetições cada, totalizando 68 parcelas, medindo 3x3 m cada. Para a distribuição das parcelas utilizou-se o delineamento experimental em bloco totalmente casualizado, com quatro repetições para cada tratamento, sendo combinação de resíduos de carvoeira, de serraria e de açougue. A seguir a identificação de cada mistura na parcela de solo:

\section{1 - Carvão}

2 - Resíduos de pó de serra

3 - Resíduos de lâmina triturada

4 - Resíduos de ossos

5 - Carvão + Resíduos de pó de serra

6 - Carvão + Resíduos de lâmina triturada

7 - Carvão + Resíduos de ossos 
8 - Resíduos de pó de serra + Resíduos de lâmina triturada

9 - Resíduos de pó de serra + Resíduos de ossos

10 - Resíduos de lâmina triturada + Resíduos de ossos

11 - Carvão + Resíduos de pó de serra + Resíduos de lâmina triturada

12 - Carvão + Resíduos de lâmina triturada + Resíduos de ossos

13 - Resíduos de pó de serra + Resíduos de lâmina triturada + Resíduos de ossos

14 - Carvão + Resíduos de pó de serra + Resíduos de lâmina triturada + Resíduos de ossos

15 - Carvão + Resíduos de pó de serra + Resíduos de lâmina triturada + Resíduos de ossos + Sangue e gordura animal

16 - Branco

17 - Carvão + Resíduos de pó de serra + Resíduos de ossos

\section{RESULTADOS E DISCUSSÃO}

Os resultados estão mostrados na figura 1, onde as análises químicas da TPN determinaram as propriedades químicas de relevância para avaliação da qualidade do experimento, relacionadas aos elementos químicos disponíveis e aos atributos do solo.

Figura 1 - Resultados das análises de TPN dos parâmetros de pH, H + Al, SB, t, T, V, m, P-rem e da disponibilidade dos elementos P, K, Mg, Ca e Al, para a $1^{\mathrm{a}}$ (2004) e a $6^{\mathrm{a}}$ coleta (2011). Fonte: Própria.

$\mathrm{pH}-1^{\mathrm{a}}$ Coleta

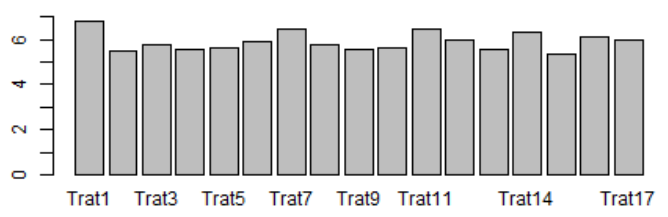

pH- $6^{a}$ Coleta

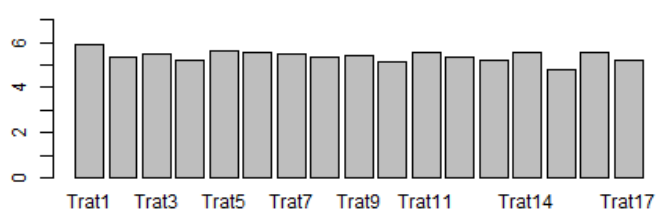

P..mg.dm ${ }^{3}$.- $1^{a}$ Coleta

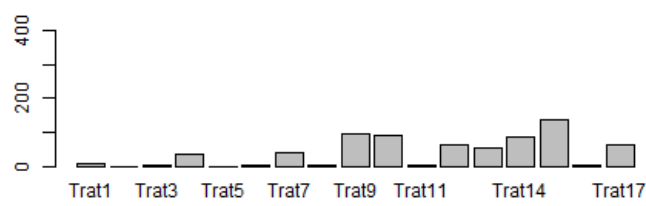

P..mg.dm ${ }^{3} .6^{a}$ Coleta

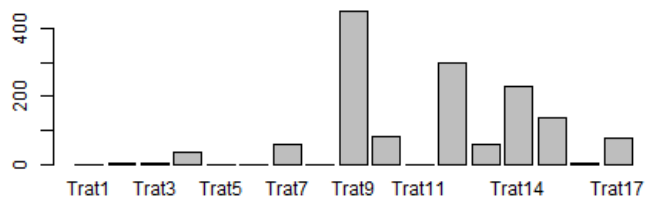


K..mg.dm ${ }^{3} .-1^{a}$ Coleta

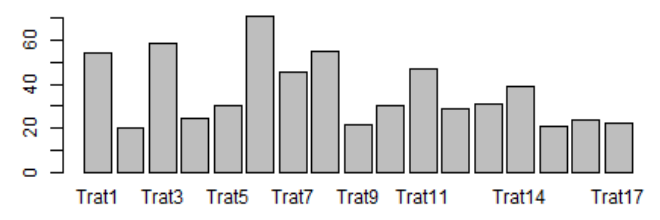

K..mg.dm ${ }^{3} .-6^{a}$ Coleta

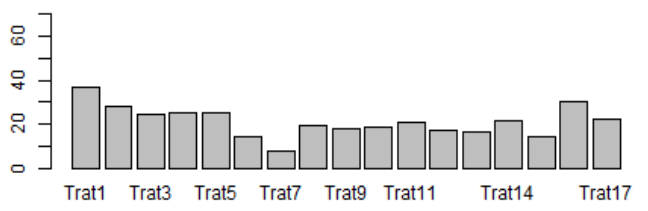

H...Al..cmolc.dm ${ }^{3}$-- $1^{\mathrm{B}}$ Coleta

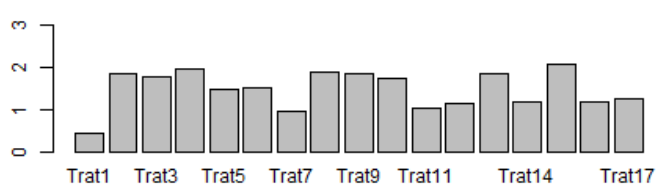

\section{H...Al..cmolc.dm ${ }^{3} .-6^{\mathrm{a}}$ Coleta}

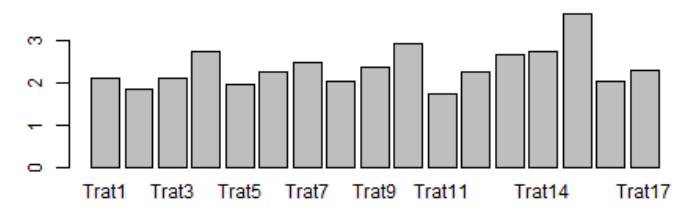

$\mathrm{Mg}^{2} . . \mathrm{cmolc} . \mathrm{dm}^{3} .-1^{\mathrm{A}}$ Coleta

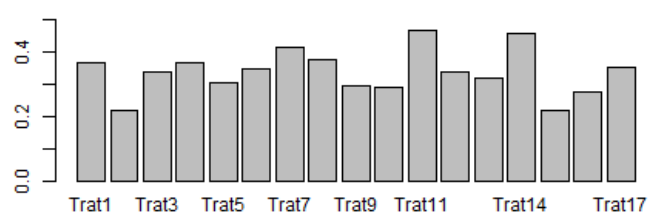

$\mathrm{Mg}^{2} . . \mathrm{cmolc} . \mathrm{dm}^{3}{ }^{3}-6^{\mathrm{a}}$ Coleta

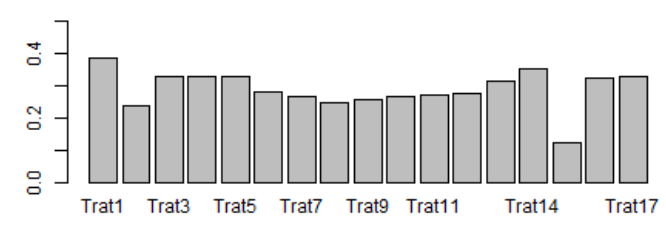

$\mathrm{Ca}^{2}$...cmolc.dm ${ }^{3}-1^{\mathrm{a}}$ Coleta

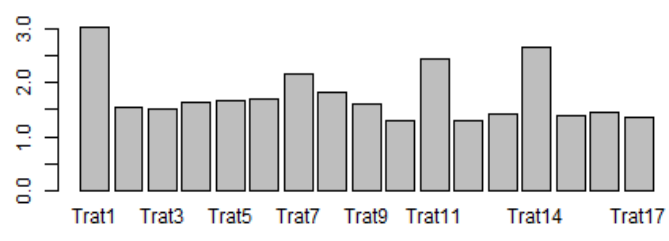

$\mathrm{Ca}^{2}$...cmolc.dm ${ }^{3} .6^{\mathrm{a}}$ Coleta

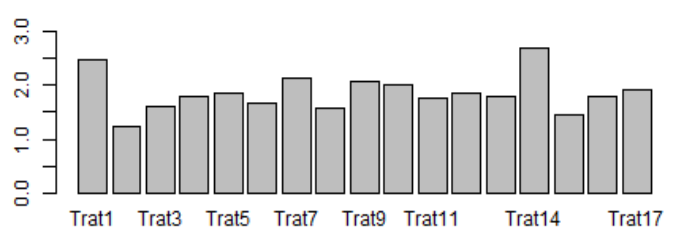

SB..cmolc.dm ${ }^{3}$.- $1^{\text {a }}$ Coleta

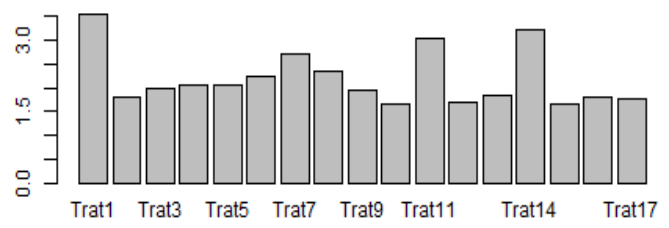

SB..cmolc.dm ${ }^{3} \cdot-6^{a}$ Coleta

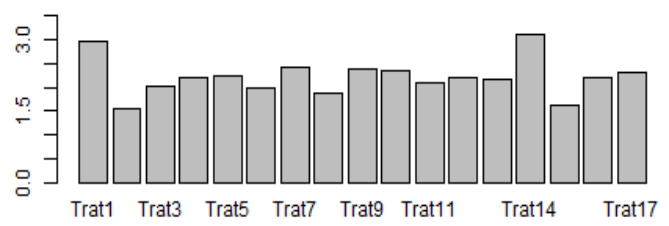

$\mathrm{Al}^{3}$...cmolc.dm ${ }^{3}$.- $1^{\text {a }}$ Coleta

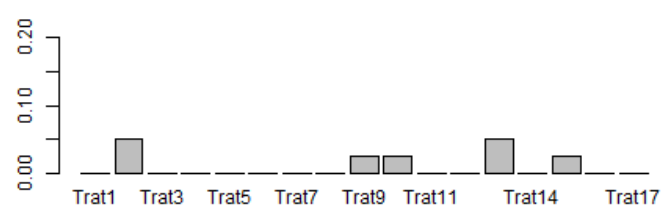

Al ${ }^{3}$...cmolc.dm ${ }^{3}$.- $6^{a}$ Coleta

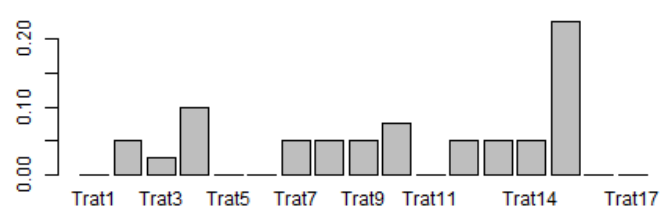




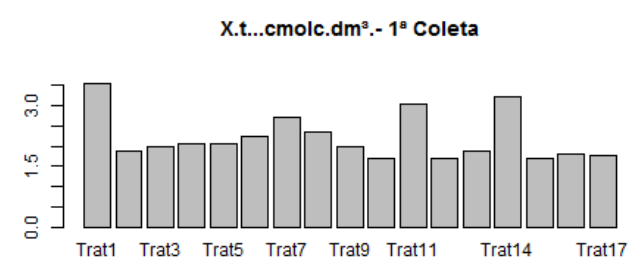

X.t...cmolc.dm ${ }^{3} .-6^{a}$ Coleta

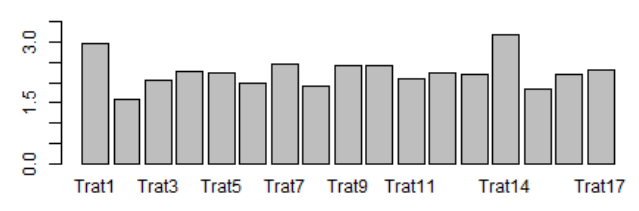

V....- $1^{\mathrm{a}}$ Coleta

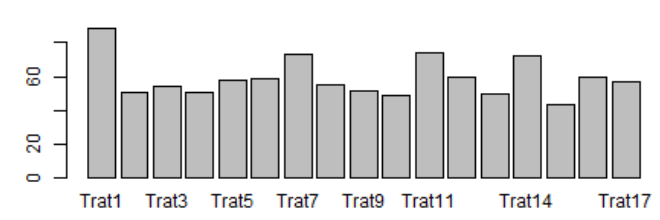

V....- $6^{a}$ Coleta

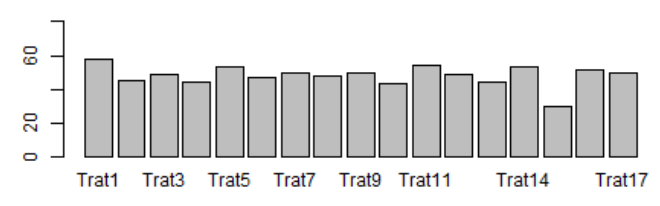

P.rem..mg.L.- $1^{\mathrm{a}}$ Coleta

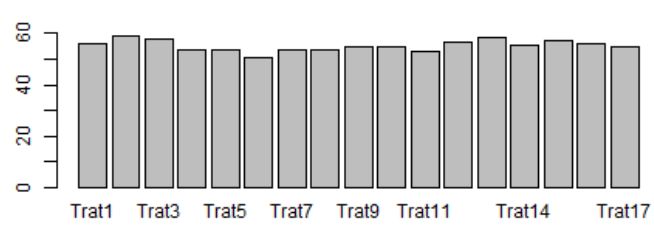

P.rem..mg.L.- $6^{a}$ Coleta

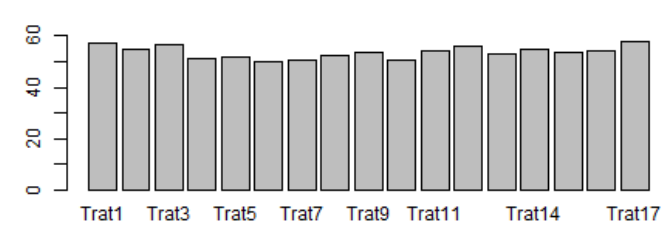

X.T...cmolc.dm ${ }^{3}$.- $1^{\text {a }}$ Coleta

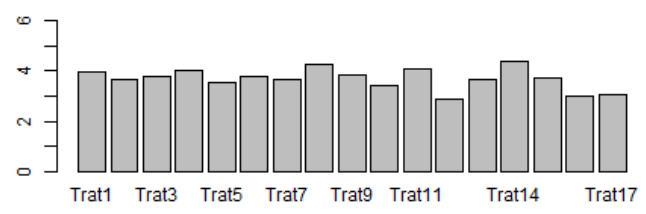

X.T...cmolc.dm ${ }^{3} \cdot 6^{a}$ Coleta

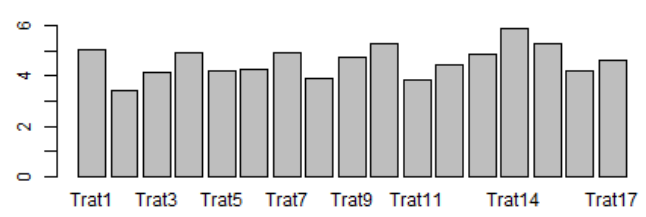

m....- $1^{\mathrm{A}}$ Coleta

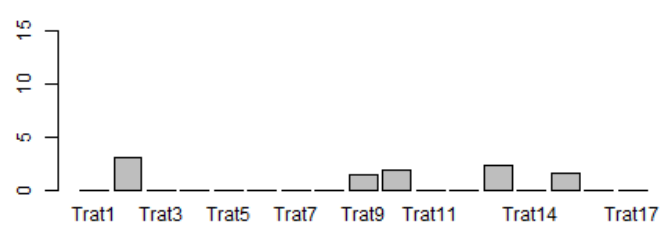

m....- $6^{a}$ Coleta

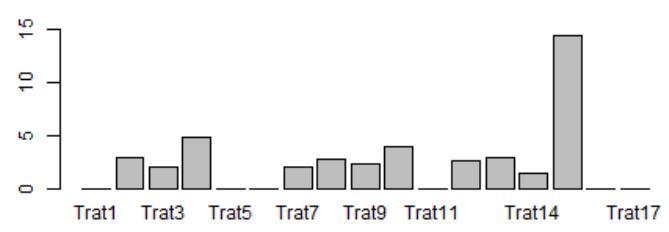

Os efeitos positivos com relação as propriedades do experimento TPN foram 
analisados com relação aos diferentes tratamentos e no intervalo de sete anos, entre a coleta do ano de 2004 e de 2011. O pH (potencial hidrogeniônico) denota a quantificação da soma dos cátions básicos e cátions ácidos da solução do solo, quanto maior a acidez do solo indica a maior proporção de cátions ácidos (EMBRAPA, 1998). O pH avaliado foi o parâmetro que menos sofreu alteração durante os períodos de avaliação, entre todos os tratamentos e entre os períodos de coleta

A acidez potencial está presente nos colóides do solo constituída de dois íons de H+ e $\mathrm{Al}^{+3}$ é utilizada para determinar a CTC efetiva. A origem do $\mathrm{H}+\mathrm{Al}$ se dá, geralmente, pela intensa lixiviação dos nutrientes do solo (EMBRAPA, 1998). Os resultados para a acidez potencial, para o alumínio e para a percentagem de saturação por alumínio (m), demonstram que houve um acréscimo nesses parâmetros, principalmente com o passar do tempo. Parte do Al complexado pode ser disponibilizado às plantas, sobretudo em condições elevadas de acidez. Com a oxidação da MO, ocorre passagem de formas de $\mathrm{Al}$ fortemente complexadas para formas mais reativas, o que pode acarretar aumento na liberação de Al para a solução do solo.

Os teores de cálcio avaliados nos tratamentos indicaram uma possível incorporação ou agregação desse mineral no solo, pois ocorreu a diminuição no parâmetro observado, da mesma forma para o potássio e o magnésio. O Ca é um macronutriente necessário para o crescimento radicular das plantas e fator essencial para a diminuição da acidez no solo, sendo muito utilizado na forma de calcário, gesso para equilíbrio do $\mathrm{pH}$ e como fertilizante (MARIA et al., 1993). O magnésio é um nutriente essencial para a nutrição de plantas da forma que faz parte do processo produção de carboidratos (LUENGO et al., 2018). O potássio é um nutriente mineral primário fornecido pelo solo usado em grandes quantidades pelas plantas (EMBRAPA, 1998).

Os resultados de Capacidade de Troca de Cátions efetiva ( $\mathrm{t}$ ) e da Capacidade de Troca de Cátions a pH 7,0 (T) apresentaram valores satisfatórios para todos os tratamentos, com destaque para os tratamentos 14 e 15. Em solos de regiões tropicais, como é o caso da região onde o experimento é desenvolvido, e há predomínio de minerais de baixa CTC, o manejo de materiais orgânicos de diversas fontes é de importância fundamental na contribuição de até 90\% de incremento na CTC do solo (ABREU JR. et al., 2001).

\section{CONCLUSÕES}

Pode-se concluir que o tratamento 14 composto pela mistura de Carvão + Resíduos de pó de serra + Resíduos de lâmina triturada + Resíduos de ossos foi o que obteve valores mais 
elevados com relação aos parâmetros analisados. Entretanto, os demais tratamentos podem ser utilizados com eficiência para diversas necessidades de correção e melhoria de solos para o cultivo das culturas desejadas pelos produtores.

\section{REFERÊNCIAS}

BACHIEGA, C. F. Z.; FERRACCIÚ, R. L. A.; FÁVERO, E. C. Teores de alumínio trocável e não trocável após calagem e gessagem em Latossolo sob sistema plantio direto. Campinas: Brangantia., 2007.

BACHIEGA, C. F. Z.; FERRACCIÚ, R. L. A.; FÁVERO, E. C. Teores de alumínio trocável e não trocável após calagem e gessagem em Latossolo sob sistema plantio direto. Campinas: Brangantia., 2007.

CAIRES, E. F.; CHUEIRI, W. A.; MADRUGA, E. F.; FIGUEIREDO, A. \&. Alterações de características químicas do solo e resposta da soja ao calcário e gesso aplicados na superfície em sistema de cultivo sem preparo do solo. Ponta Grossa: R. Bras. Ci. Solo, 1998.

COSTA, J. A.; COSTA, M. L. da; KERN, D. C. Analysis of the spatial distribution of geochemical signatures for the identification of prehistoric settlement patterns in ADE and TMA sites in the lower Amazon Basin. Journal of Archaeological Science 40, p. 2771-2782. 2013.

GERMAN, L. A. Historical contingencies in the coevolution of environment and livelihood: contributions to the debate on Amazonian Black Earth. Geoderma 111307 $331,2003$.

GLASER, B.; HAUMAIER, L.; GUGGENBERGER, G.; ZECH, W. The "Terra Preta" phenomenon: a model for sustainable agriculture in the humic tropics. Naturwissenschafter, v. 88, p. 37-41, 2001.

JR, C. H. A.; MURAOKA. T.; OLIVEIRA. F. C. Cátions trocáveis, capacidade de troca de cátions e saturação por bases em solos brasileiros adubados com composto de lixo urbano. Piracicaba: Scientia Agricola, 2001.

KERN, D. C.; COSTA, J. A.; AQUINO, G. D. ; KAMPF, N. ; COSTA, M. L.; RUIVO, M. L. P. ; LEMOS, V. P.; Costa, Marcondes Lima da; Lemos, V.P. Ocupação humana pré-colonial no sitio Ilha de Terra, Caxiuanã. In: Pedro Luis Braga Lisboa. (Org.). Caxiuanã paraíso ainda preservado. 1ed. Belém: Núcleo Editorial de Livros, 2013, v. 1, p. 21-654.

KERN, D.C. Geoquimica e pedogeoquimica de sitios arqueológicos com Terra Preta na Floresta Nacional de Caxiuaná (Portel-Pará). Belém: Universidade Federal do Pará, 1996. (Tese de Doutorado), 1996. 124p.

KERN, D.C. Geoquímica e pedogeoquímica de sítios arqueológicos com Terra Preta na Floresta Nacional de Caxiuanã (Portel-Pará). Belém: Universidade Federal do Pará, 1996. (Tese de Doutorado), 1996. 
LUENGO, R. F., Butruille, N.-M. d., \& Melo, R. d. Determinação de minerais no solo e análise de folhas de couve produzida em Brasília. Brasília: Brazilian Journal of food technology, 2018.

MARIA, I. C.; ROSSETTO, R.; AMBROSANO, E. J.; CASTRO, O.M. Efeito da adição de diferentes fontes de cálcio no movimento de cátions em colunas de solo. Piracicaba: Sci. Agric, 1993.

NOVOTNY, E. H.; HAYES, M. H. B.; MADARI, B. E.; et al. Lessons from the Terra Preta de Indios of the Amazon region for the utilisation of charcoal for soil amendment, J. Braz. Chem. Soc. 20, 1003-1010, 2009.

SILVEIRA, I. M., KERN, D. C., QUARESMA, H. D. A. B. Flona Caxiuanã: reconstituição de uma ocupação In: LISBOA, P.L.B. Caxiuanã: populações tradicionais, meio físico \& diversidade biológica. 59-76p. 2002.

TEIXEIRA, W. G.; KERN, D. C.; MADARI, B.; LIMA, H. N.; WOODS, W. As Terras Pretas de Índio da Amazônia: Sua Caracterização e Uso deste Conhecimento na Criação de Novas Áreas. Publisher: Embrapa Amazônia Ocidental, Manaus, AM, 358 p., 2009.

THEODORO, V. C. A.; ALVARENGA, M. I. N.; GUIMARÃES, R. J.; SOUZA, C. A. S. Alterações químicas em solo submetido a diferentes formas de manejo do cafeeiro. Lavras, 2003. 